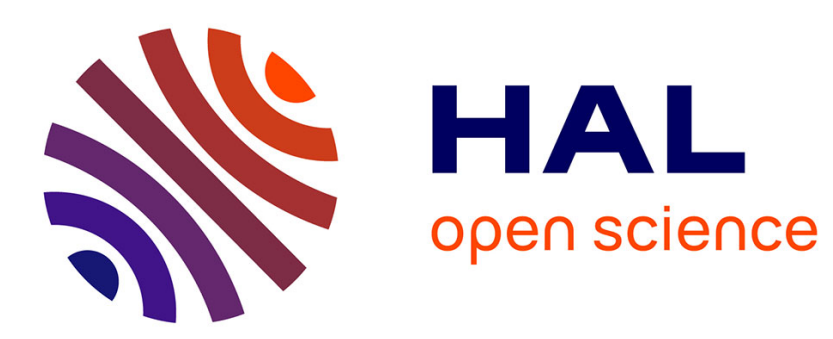

\title{
Demo Abstract: Spatial modulation based transmission using a reconfigurable antenna
}

\author{
Yvan Kokar, Kammel Rachedi, Abdel Ourir, Julien de Rosny, Dinh-Thuy \\ Phan-Huy, Jean-Christophe Prévotet, Maryline Helard
}

\section{- To cite this version:}

Yvan Kokar, Kammel Rachedi, Abdel Ourir, Julien de Rosny, Dinh-Thuy Phan-Huy, et al.. Demo Abstract: Spatial modulation based transmission using a reconfigurable antenna. 2019 IEEE INFOCOM, Apr 2019, Paris, France. hal-02124126

\section{HAL Id: hal-02124126 \\ https://hal.science/hal-02124126}

Submitted on 9 May 2019

HAL is a multi-disciplinary open access archive for the deposit and dissemination of scientific research documents, whether they are published or not. The documents may come from teaching and research institutions in France or abroad, or from public or private research centers.
L'archive ouverte pluridisciplinaire HAL, est destinée au dépôt et à la diffusion de documents scientifiques de niveau recherche, publiés ou non, émanant des établissements d'enseignement et de recherche français ou étrangers, des laboratoires publics ou privés. 


\title{
Demo Abstract: Spatial modulation based transmission using a reconfigurable antenna
}

\author{
Y. Kokar ${ }^{1}$, K. Rachedi ${ }^{2}$, A. Ourir ${ }^{2}$, J. de Rosny ${ }^{2}$, D.-T. Phan-Huy ${ }^{3}$, J.-C. Prévotet ${ }^{1}$, M. Hélard ${ }^{1}$ \\ ${ }^{1}$ Univ. Rennes, INSA Rennes, IETR, CNRS, UMR 6164, Rennes, France \\ ${ }^{2}$ ESPCI Paris, PSL University, CNRS, Institut Langevin, Sorbonne Université, Paris, France \\ ${ }^{3}$ Orange Labs, Châtillon, France
}

\begin{abstract}
This demonstration presents a spatial modulation based scheme between a transmitter connected to a compact reconfigurable antenna and a receiver connected to 4 omnidirectional antennas. The reconfigurable antenna used to perform spatial modulation is able to generate 8 uniformly distributed radiation patterns. First, a Radio Wave Display will show the 8 radiation patterns of the reconfigurable antenna. In a next step, the BER will be displayed after spatial demodulation at the receiver side to evaluate the communication performance.

Index Terms-5G, spatial modulation, Internet of Things
\end{abstract}

\section{INTRODUCTION}

The fifth generation of mobile network (5G) will become the backbone of the Internet of Things (IoT). Among the different studied technologies for IoT, Spatial Modulation (SM) MIMO appears as a good candidate for a transmission from a connected object to an access point. Indeed, by taking benefit from spatial diversity, SM-MIMO provides a high data rate while leading to low complexity and low power connected objects.

SM-MIMO may transmit two distinct data bit streams per symbol. The first is transmitted trough a conventional modulation, while the second bit stream is sent by selecting one antenna among the $N_{t}$ antennas of the transmitter, at each symbol period. Each transmit antenna is thus associated to a bit sequence value of length $\log _{2}\left(N_{t}\right)$.

Increasing $N_{t}$ leads to improve the data rate of the SM stream. Unfortunately, a large number of antennas cannot be easily implemented in small connected objects. To circumvent this issue, the use of a compact reconfigurable antenna has been proposed in [1]. A reconfigurable antenna is capable of selecting one Radiation Pattern (RP) among $N_{t}$ available. Thus, each RP may now be associated to a different bits sequence.

In [2], a SM-MIMO 2x2 testbed, which uses a RF chain per transmit antenna ( $2 \mathrm{RF}$ in total), has been presented. In this demonstration, we present a SM-MIMO testbed with only one RF chain at the transmitter side. The unique RF chain feeds a reconfigurable antenna which is capable of generating 8 distinct RP.

\section{TESTBED DESCRIPTION}

As illustrated in Fig. 1, the proposed testbed is composed of two WARP v3 boards and a reconfigurable antenna. One

The authors would like to thank the SPATIAL MODULATION project funded by the French National Research Agency (ANR).

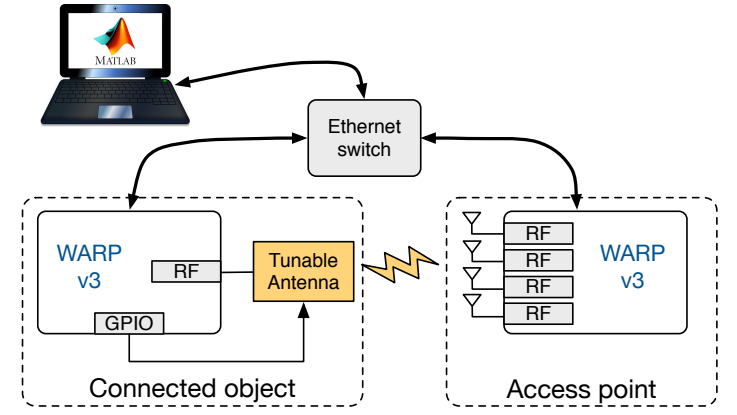

Fig. 1. Testbed description

WARP board acts as a Tx transmitter (representing the connected object) and feeds the reconfigurable antenna which is controlled by the GPIO pins of the WARP board. The other WARP board acts as a $\mathrm{Rx}$ receiver (representing the access point) and is connected to four omnidirectional antennas. Both WARP boards are connected to an external workstation via an Ethernet switch. Tx and Rx baseband processing is performed in software, on the workstation.

\section{A. The reconfigurable split ring antenna}

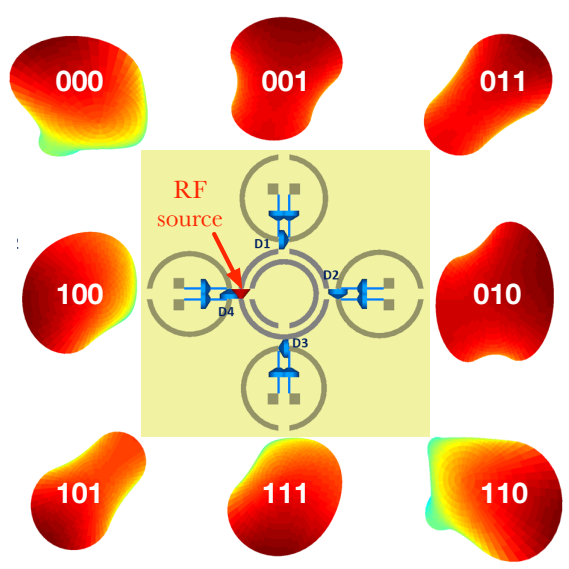

Fig. 2. The SRR antenna and its 8 radiation patterns

The main element of the reconfigurable SRR antenna shown in Fig. 2 is a double closed rings. In order to deliver different $\mathrm{RP}$, the double closed rings is surrounded by four parasitic 
loops whose resonant frequency is controlled by a pin diode (in blue) placed in the gap of the loop. By controlling the modes of the 4 pin diodes (ON or OFF), the SRR antenna is able to steer in 8 uniformly distributed directions as depicted in Fig. 2. Each RP is associated to 3 bits values which will be transmitted by the SM. The RP mapping is done such that only one bit differs for 2 adjacent RP.

\section{B. Baseband processing}

As shown in Fig. 3, the structure of the transmitted frame is composed of 4 parts which correspond to the 4 phases required for SM based transmission. The first phase is to

\begin{tabular}{|l|l|l|l|}
\hline AGC seq. & Sync. seq. & Pilot Symbols & Data Symbols \\
\hline
\end{tabular}

Fig. 3. Structure of transmitted frame

determine, thanks to a transmitted Automatic Gain Control (AGC) sequence, the appropriate gain at the receiver which suits for all SRR antenna RP. The next phase is to accurately evaluate the beginning of the pilot and data symbols. To that end, a synchronization sequence is inserted in the transmitted frame before the pilot symbols and a cross-correlation is performed at the receiver between the received signal and the synchronization sequence. Pilot symbols are then used in order to estimate the channels between each SRR antenna RP and the receiver antennas. Data symbols are generated trough a conventional modulation, and SM is performed by selecting the RP (via GPIO pins) corresponding to the 3 SM bits value that have to be transmit on the current sent symbol. At the receiver side, spatial demodulation consists in detecting, for each received symbol, which RP has sent the current symbol in order to retrieve the SM bits. To that end, we use the maximum likelihood detector as described in [2].

\section{DEMONSTRATION}

In this demonstration, we will present a transmit SM based wireless transmission. The setup of our demonstration is depicted in Fig. 4. The carrier frequency and the bandwidth

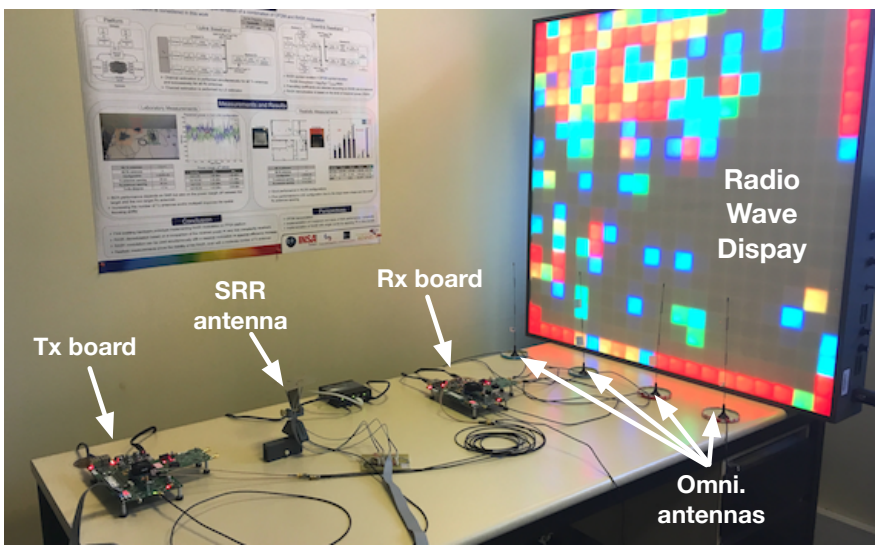

Fig. 4. Demonstration setup of the transmission are respectively set to $2.45 \mathrm{GHz}$ and 40 $\mathrm{MHz}$.

The demonstration is conducted in two steps. In a first step, we show to participants how it is possible to visually distinguish the 8 different RP of the SRR antenna. For this purpose, we use the Radio Wave Display (RWD) [3] as a real time receiver which allows to display the radiation of a transmitting antenna that is placed in front of it. The RWD comprises 400 (20 by 20) power sensors operating between $50 \mathrm{MHz}$ and $3 \mathrm{GHz}$. The power measured by each sensor is then converted into a gradient of colors following a thermal color scale. Fig. 5 provides pictures of the RWD for the 8 RP of the SRR antenna corresponding to the bits transmitted using SM. We can clearly observe that the 8 patterns of the SRR antenna are visually distinct.
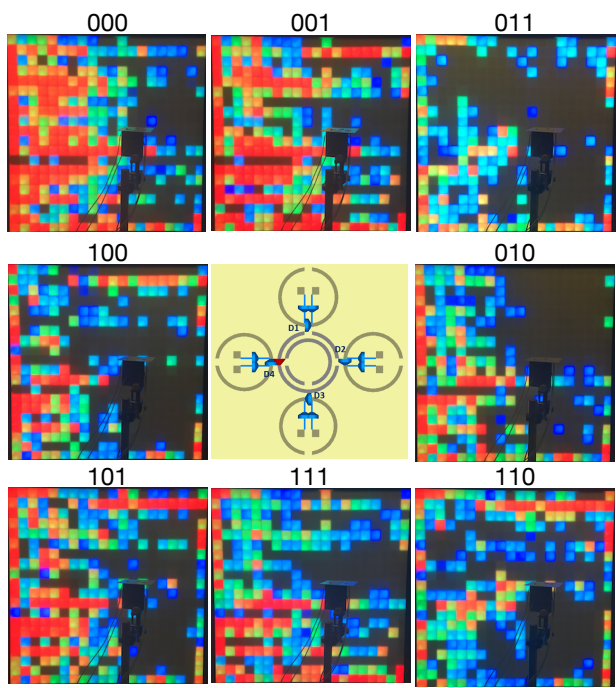

Fig. 5. Visualisation of the SRR antenna radiation patterns on the RWD

In a second step of the demonstration, we will perform a transmission of 900 data bits using SM between the transmitter board (acting as the connected object) and the receiver board (representing the access point). After the demodulation of the received frame, the Bit Error Rate (BER) of the transmission is displayed on the workstation. Participants are then be able to observe the effect of changing the SRR antenna and/or receiving antennas positions (few centimetres to few meters) on the quality of the communication (BER).

A demonstration video is available online in [4].

\section{REFERENCES}

[1] A. Ourir, K. Rachedi, D. . Phan-Huy, C. Leray, and J. de Rosny, "Compact reconfigurable antenna with radiation pattern diversity for spatial modulation," in 2017 11th European Conference on Antennas and Propagation (EUCAP), March 2017, pp. 3038-3043.

[2] N. Serafimovski, A. Younis, R. Mesleh, P. Chambers, M. D. Renzo, C. Wang, P. M. Grant, M. A. Beach, and H. Haas, "Practical implementation of spatial modulation," IEEE Transactions on Vehicular Technology, vol. 62, no. 9, pp. 4511-4523, Nov 2013.

[3] http://www.luxondes.com/en/an_produits_dalle400/.

[4] https://youtu.be/L4-_UonrHGU. 